\title{
Comment je suis devenu une drag queen*
}

\section{Eberhard Wolff}

Prof. Dr rer. soc., rédacteur culture, histoire, société

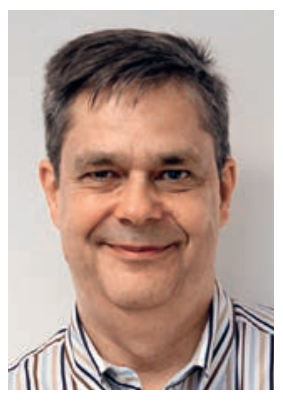

Nous avons entendu cette histoire à maintes reprises. En partant de Miami, Haroldo Rodriguez Danhakl s'est épilé les sourcils. Puis il s'est rasé les jambes. Il était passé dans l'autre genre. Elle est finalement arrivé à New York avec l'identité de Holly Woodlawn.

La chanson de Lou Reed, Walk on the Wild Side, commence par: «Holly came from Miami F.L.A....». Elle date de 1972. A l'époque, les personnes dont l'identité sexuelle n'était pas conventionnelle vivaient cachées, avaient mal tourné ou basculé dans l'avant-garde artistique. Aux yeux des personnes dites «normales», ces individus étaient au mieux déboussolés, comme Lola pour le chanteur des «Kinks», dans sa chanson éponyme de 1970. On avait à la rigueur vu des jambes d'hommes rasées dans des comédies basées sur les quiproquos, comme Certains l'aiment chaud en 1959, avec Tony Curtis et Jack Lemmon.

En novembre dernier, la Cour constitutionnelle fédérale allemande a demandé aux autorités de permettre l'enregistrement d'un troisième genre, ou de renoncer totalement aux indications de genre, afin de préserver les droits de la personnalité des personnes intersexuées et de celles dont l'identité sexuelle diffère du genre biologiquement défini.

Dans un article très éclairant [1], Stefan Hirschauer, sociologue allemand, spécialisé dans l'étude des genres, estime à ce sujet que dans les sociétés modernes, le fossé entre les attributions claires de genre est de plus en plus large. La catégorie "Nationalité» devient elle aussi de plus en plus floue et perd de l'importance chez nous, déclare S. Hirschauer.

«He» ou «She», la distinction n'est plus aussi nette qu'à l'époque des paroles de Lou Reed. Les frontières entre les genres sont de plus en plus perméables. Ceux qui travaillent dans le secteur de la santé sont confrontés plus tôt que les autres à cette réalité. Mais ces profondes mutations culturelles n'apparaissent pas d'un coup de baguette magique. Elles s'imposent dans notre quotidien à travers de modestes évolutions.

Cette année, dans la célèbre émission télévisée Germany's Next Top Model, un groupe de "drag queens" montrait aux jeunes mannequins comment bien se mettre en scène. Il ne fait aucun doute que les hommes modelés en superfemmes sont des "oiseaux exotiques» à l'allure totalement extravagante, arborant les clichés de la féminité, mais ils sont moins qu'avant mis au ban de la société. Ils sont aussi admirés, voire inspirent les futures modèles pour leur performance chez Heidi Klum et les millions de personnes qui la suivent sur les différents médias.

Le dernier Zurich Pride Festival en faveur de la diversité des sexes et des genres n'était plus autant centré sur la défense des minorités opprimées. Sponsorisé par des établissements aussi reconnus qu'UBS, Credit Suisse et la Poste, ainsi que par les départements «Queer» des CFF, des VBZ et de la police municipale, ou par des partis comme le Parti Bourgeois Démocratique Suisse (PBD), il est en passe de devenir un événement consacré en termes de diversité. Dans vingt ans, ce sera peut-être le nouveau «Sechseläuten».

Mais les profondes mutations culturelles, comme la perméabilité croissante des frontières entre les genres, se reflètent également dans un quotidien encore plus tangible. De plus en plus souvent, les hommes adoptent un style de coiffure qui aurait été auparavant jugé comme typiquement féminin. Ils n'ont plus besoin désormais de faire clandestinement partie d'une formation musicale féminine pour se faire raser les jambes. Pour nombre de cyclistes, les jambes glabres sont devenues une sorte de signe de reconnaissance. Certes, c'est utile pour l'aérodynamisme, les massages ou la guérison des blessures. Mais quand on parle d'une meilleure mise en évidence des muscles péroniers, il s'agit déjà d'esthétique.

Chignons et queues-de-cheval sont désormais courants chez les hommes. A moins que ce ne soit déjà un signe de la nouvelle virilité, comme la barbe, très branchée.

Peut-être s'agit-il plutôt d'une intensification du soin que les hommes prennent de leurs poils. Aujourd'hui, de plus en plus d'hommes se rasent sous les aisselles, alors qu'au contraire les femmes à la mode se laissent pousser les poils à cet endroit.

Personnellement, je ne porte pas de chignon et je ne me suis jamais rasé les jambes. Ni ne pratique le cyclisme. Pourtant, depuis la chanson Walk on the Wild Side, les changements ont aussi laissé de légères traces dans mon quotidien. Depuis quelques années, je me rase les aisselles en été. Chaque fois, je me rapproche un peu plus de Holly, figure normale et quotidienne. Mon identité sexuelle établie n'en est pas perturbée pour autant. 